\title{
Fluctuations in a mesoscopic superconducting ring: resonant behavior of conductivity and specific heat in two mode critical regime
}

\author{
S.V. Mironov ${ }^{1}$ and A. Buzdin ${ }^{2}$ \\ ${ }^{1}$ Institute for Physics of Microstructures, Russian Academy of Sciences, 603950 Nizhny Novgorod, GSP-105, Russia \\ ${ }^{2}$ Institut Universitaire de France and University Bordeaux, \\ LOMA UMR-CNRS 5798, F-33405 Talence Cedex, France
}

(Dated: December 6, 2018)

\begin{abstract}
The critical fluctuations in a mesoscopic superconducting ring are studied within the GinzburgLandau approach. The nonlocal conductivity as well as the specific heat are calculated as functions of the magnetic flux $\Phi$ through the ring. At $\Phi=\Phi_{0} / 2$ two low-energy eigenstates become degenerate and near this point the behavior of fluctuations-dependent quantities change dramatically: both the zero Fourier component $\sigma_{0}$ of the fluctuation conductivity and the specific heat become nonmonotonic functions of $\left|\Phi-\Phi_{0} / 2\right|$ with rather special resonant structure.

PACS numbers: 74.25.F-, 74.78.Na, 72.10.-d, 71.10.Ay, 71.30.+h.
\end{abstract}

\section{INTRODUCTION}

Mesoscopic superconducting rings attract considerable interest in connection with their unusual properties in the vicinity of the superconduction transition. One of the most interesting features is an oscillatory behavior of the critical temperature as a function of magnetic flux through the ring $\frac{1}{\underline{1}}$ due to competition between spatial modes characterizing by different orbital momenta. The remarkable property of the superconducting rings is the fact that all eigenstates are well separated from each other and in some cases the dynamics of each state can be described independently. This provide a unique opportunity to study the critical fluctuation contribution to all measurable quantities of the rings. With the development of the microfabrication technologies it becomes possible to create superconducting rings of the radius $R$ comparable to the Ginzburg-Landau coherence length $\xi_{0}$ (see Refs. 2,3 and references therein). The fluctuations in such small rings can be described in the frames of simple analytical models, such as the zero-dimensional Ginzburg-Landau formalism (see, for example, Ref. 4). In Refs. 5, 6 the persistent current due to the critical fluctuations was studied for different ratio between the rings' radius and the coherence length. The calculation of magnetization in the critical regime have been performed in Ref. 7. Note that previously the influence of the critical fluctuations on specific heat and magnetization has been studied in small superconducting granula both theoretically $\stackrel{8}{\underline{8}}$ and experimentally $\stackrel{9}{\text {. }}$.

At the same time one of the most natural way to study fluctuations near $T_{c}$ is the performance of the conductivity measurements (the fluctuation correction to the conductivity has been described first in Ref. 10). For superconducting rings two types of experiments were proposed: (i) nonlocal paraconductivity measurements with probes attached to different points of the ring ${ }^{11.12}$; (ii) contactless measurements of dissipation in the array of rings subject to an electromotive force induced by a weak alternating magnetic field 13 . The corresponding calcula- tions of the nonlocal conductivity within the Gaussian approximation were performed in Ref. 14 .

The separation of different eigenstates in mesoscopic ring with $R \gtrsim \xi_{0}$ allows to describe analytically the contribution of the critical fluctuations to the nonlocal conductivity in magnetic field which produces the flux $\Phi$ through the ring. It was shown that in the critical fluctuation regime the fluctuation contribution to the conductivity has a logarithmic singularity near the critical temperature $T_{C} \underline{13}$ with a prefactor proportional to the $\left(\Phi / \Phi_{0}\right)^{2}$. However this approach is valid only for magnetic flux values which are not close to $\Phi_{0} / 2$. Otherwise for $\Phi \approx \Phi_{0} / 2$ the separation of modes with different orbital momenta breaks down: critical fluctuations near $T_{c}$ are produced by two interacting modes, which can not be considered separately anymore.

In the present paper we suggest an analytical description of critical fluctuations for the case $\Phi \approx \Phi_{0} / 2$ and calculate the corresponding fluctuation correction to the non-local conductivity of the ring. We will keep in mind the contactless realization of conductivity measurements in which only the zero Fourier component of the conductivity plays the key role. Also we analyze the behavior of the specific heat value in two-mode critical regime. To analyze the situation we use time-dependent GinzburgLandau equation. This approach is reasonable for small dirty superconducting rings (see Ref. 13).

The paper has the following structure. In Section $\amalg$ we describe in detail the behavior of the conductivity both inside and outside of the two-mode critical regime. In particular, we present the exact expression for the conductivity for the case $\phi \approx 1 / 2$, based on the analytical solution of nonlinear two-mode Ginzburg-Landau equation. In Section [II we calculate the specific heat taking the mode interaction into account. The results are summarized in Section IV. 


\section{FLUCTUATION CONDUCTIVITY DUE TO CRITICAL FLUCTUATIONS}

To calculate the non-local conductivity we use the approach which is similar to that of Ref. 13. The zerofrequency conductivity $\sigma\left(\varphi-\varphi^{\prime}\right)$ is given by the Kubo formula (see, for example, Ref. 14)

$$
\sigma\left(\varphi-\varphi^{\prime}\right)=\frac{1}{T} \int_{0}^{\infty}\left\langle J(\varphi, 0) J\left(\varphi^{\prime}, t\right)\right\rangle d t
$$

where the supercurrent is defined by the standard expression $J(\varphi, t)=(e / m R) \operatorname{Re}\left[\psi\left(i \partial_{\varphi}-\phi\right) \psi^{*}\right]$ and $\phi=\Phi / \Phi_{0}$.

Performing the Fourier transform of the order parameter wave function and the conductivity

$$
\psi(\varphi, t)=\sum_{n} \psi_{n}(t) e^{i n \varphi}, \sigma\left(\varphi-\varphi^{\prime}\right)=\sum_{k} \sigma_{k} e^{i k\left(\varphi-\varphi^{\prime}\right)}
$$

one can obtain the following expression for the zero Fourier component $\sigma_{0}$

$$
\begin{aligned}
& \sigma_{0}=\frac{4}{T}\left(\frac{e}{2 m R}\right)^{2} \int_{0}^{\infty} d t \sum_{n, m}(n-\phi)(m-\phi) \\
& \times\left\langle\left|\psi_{n}(0)\right|^{2}\left|\psi_{m}(t)\right|^{2}\right\rangle,
\end{aligned}
$$

while for nonzero components $\sigma_{k \neq 0}$

$$
\begin{aligned}
\sigma_{k}=\frac{1}{T} & \left(\frac{e}{2 m R}\right)^{2} \int_{0}^{\infty} d t \sum_{n}(2 n+k-2 \phi) \\
& \times\left\langle\psi_{n}^{*}(0) \psi_{n}(t) \psi_{n+k}(0) \psi_{n+k}^{*}(t)\right\rangle .
\end{aligned}
$$

The Fourier components of the order parameter wave function $\psi_{n}(t)$ satisfy the non-linear time-dependent Ginzburg-Landau equation 15

$$
-\gamma \partial_{t} \psi_{n}=a(T) \psi_{n}+b \sum_{k+m-p=n} \psi_{k} \psi_{p}^{*} \psi_{m}
$$

where the values $a=\left(4 m \xi_{0}^{2}\right)^{-1}\left[\varepsilon+\left(\xi_{0} / R\right)^{2}(n-\phi)^{2}\right]$ and $b$ are the parameters of the Ginzburg-Landau theory, $\varepsilon=$ $\left(T-T_{c 0}\right) / T_{c 0}, \xi_{0}^{-2}=4 m \alpha T_{c 0}$ and $\gamma=\pi \alpha / 8$.

Since the critical temperature $T_{c}(\phi)$ is a periodic function with the period 1 due to the Little-Parks effect and $T_{c}(-\phi)=T_{c}(\phi)$ we can consider only the range $0 \leq \phi \leq 1 / 2$ for the magnetic flux. In this range $T_{c}(\phi)=\bar{T}_{c 0}\left[1-\left(\xi_{0} / R\right)^{2} \phi^{2}\right]$ and it is convenient to use the parameter $\varepsilon^{*}=\left[T-T_{c}(\phi)\right] / T_{c 0}=\varepsilon+\left(\xi_{0} / R\right)^{2} \phi^{2}$ instead of $\varepsilon$.

Let us analyze the dependencies of the conductivity on the magnetic flux through the ring in different temperature ranges. In case when when $\varepsilon^{*} \gg G i_{(0)}$ the system is in the Gaussian regime and one can neglect the nonlinearity in Eq. (5). Here we have introduced the GinzburgLevanyuk number $G i_{(0)}$ characterizing the width of the critical fluctuation region in zero-dimensional systems:
$G i_{(0)}=\sqrt{2 b / \alpha^{2} T_{c} V}$, where $V=2 \pi R s$ is the volume of the ring and $s$ is its cross-section. In the dirty limit a simple estimate shows that $G i_{(0)} \propto\left(T_{c} / E_{F}\right)\left[\left(\xi_{0} l\right)^{3 / 4} / \sqrt{V}\right]$, where $l$ is the electron mean free path. Overwise when $\varepsilon^{*} \ll G i_{(0)}$ the fluctuations of one or two lowest modes become critical and nonlinear terms in Eq. (5) begin to play the key-role in the description of the fluctuation conductivity. Further we will consider these fluctuation regimes separately.

\section{A. Gaussian regime}

In the Gaussian regime one can find from the linearized Eq. (5) that $\psi_{n}(t)=\psi_{n}(0) \exp \left(-t / \tau_{n}\right)$, where $\tau_{n}^{-1}=\left(8 T_{c 0} / \pi\right)\left[\varepsilon^{*}+\left(\xi_{0} / R\right)^{2} n(n-2 \phi)\right]$. Also it is easy to find the exact expressions for correlators $\left\langle\left|\psi_{n}(0)\right|^{2}\right\rangle$ and $\left\langle\left|\psi_{n}(0)\right|^{4}\right\rangle$ which contribute to $\sigma_{0}$ :

$$
\begin{aligned}
\left\langle\left|\psi_{n}(0)\right|^{2}\right\rangle & =\frac{4 m \xi_{0}^{2} T}{V\left[\varepsilon^{*}+\left(\xi_{0} / R\right)^{2} n(n-2 \phi)\right]}, \\
\left\langle\left|\psi_{n}(0)\right|^{4}\right\rangle & =\frac{2\left(4 m \xi_{0}^{2}\right)^{2} T^{2}}{V^{2}\left[\varepsilon^{*}+\left(\xi_{0} / R\right)^{2} n(n-2 \phi)\right]^{2}} .
\end{aligned}
$$

From the expression (3) one can see that the singular part of $\sigma_{0}$ comes only from terms with $m=0$ and $m=1$. The corresponding expression for the fluctuation conductivity in the Gaussian regime is rather complicated and therefore is given in the Appendix. Here we will focus only on two limiting cases.

For $\varepsilon^{*} \ll\left(\xi_{0} / R\right)^{2}(1-2 \phi)$ the most singular part in the temperature dependence of the conductivity comes from the mode with $n=0$ and has the form

$$
\sigma_{0}^{(G)}\left(\varepsilon^{*}\right)=\frac{e^{2}}{4 \pi s^{2}}\left(\frac{\xi_{0}}{R}\right)^{4} \frac{\phi^{2}}{\left(\varepsilon^{*}\right)^{3}} .
$$

For $\varepsilon^{*} \gg\left(\xi_{0} / R\right)^{2}(1-2 \phi)$ in the vicinity of the half quantum flux through the ring two modes with $n=0$ and $n=1$ contribute to the most singular part of $\sigma_{0}$, which reads as

$$
\sigma_{0}^{(G)}\left(\varepsilon^{*}\right)=\frac{e^{2}}{4 \pi s^{2}}\left(\frac{\xi_{0}}{R}\right)^{4} \frac{\phi^{2}+2(1-2 \phi)^{2}}{\left(\varepsilon^{*}\right)^{3}} .
$$

These expressions are valid for any $\phi$ from the range $0 \leq$ $\phi \leq 1 / 2$.

\section{B. Critical regime with noninteracting modes}

Let us first consider critical fluctuations for magnetic flux values which are far from $\phi=1 / 2$. In this case only one spatial mode with $m=0$ is in a critical regime and thus different spatial modes do not interact with each other. Then the equation (5) for $\psi_{0}(t)$ can be 
solved analytically $\stackrel{13}{ }$. Note that the dominant contribution to the conductivity comes from the correlator with $n=m=0$. Considering only this correlator in the expression (3) (the lowest mode approximation) one can reproduce the result of Ref. 13 with corrected numerical coefficient (the correlator $\left\langle\left|\psi_{0}(0)\right|^{2}\left|\psi_{0}(t)\right|^{2}\right\rangle$ should be calculated instead of the correlator $\left\langle\psi_{0}(0) \psi_{0}^{*}(t)\right\rangle^{2}$, which provide an additional factor $\left.(8 \pi)^{1 / 2} \Gamma^{-2}(1 / 4) \approx 0.38\right)$ :

$$
\sigma_{0}^{(0)}(\phi)=\frac{e^{2}}{\pi^{3 / 2} s^{2}}\left(\frac{\xi_{0}}{R}\right)^{4} \phi^{2} \frac{1}{G i_{(0)}^{3}} \ln \frac{1}{\varepsilon^{*}} .
$$

Note that the lowest mode approximation, which has been used to obtain the expression (10), is valid when the sum of all neglected terms in (3) is small compared with $\sigma_{0}^{(0)}$. Let us calculate all terms with $m=0$ and $m=1$ in the expression (3) which can become singular near $T_{c}(\phi)$ for $0 \leq \phi \leq 1 / 2$. The corresponding expression for the conductivity $\sigma_{0}^{(C)}$ is given in the Appendix since it is rather cumbersome.

Near $\phi=1 / 2$ for $\varepsilon^{*} \ll\left(\xi_{0} / R\right)^{2}(1-2 \phi)$ the most singular correction to the conductivity (10) is negative and can be written in the form

$$
\Delta \sigma_{0}^{(0)}=-\frac{e^{2}}{8 \pi s^{2}}\left(\frac{\xi_{0}}{R}\right)^{2} \frac{1}{G i_{(0)}^{2}} \frac{1}{(1-2 \phi)} \ln \frac{1}{\varepsilon^{*}} .
$$

From the expression (11) one can see that for $|\phi-1 / 2|<\left(R / \xi_{0}\right)^{2} G i_{(0)}$ the value $\Delta \sigma_{0}^{(0)} / \sigma_{0}^{(0)}$ exceeds 1 which indicates that the expression (10) is not valid in this region. Note that in our case $R \gtrsim \xi_{0}$ and the typical value for the Ginzburg-Levanyuk number is $G i_{(0)} \sim 10^{-3}$ (for $\mathrm{Al}$ rings ${ }^{11}$ ), so the lowest mode approximation breaks down only in a narrow region near the point $\phi=1 / 2$ when both modes with $m=0$ and $m=1$ are strongly fluctuating and interact with each other. The case when $|\phi-1 / 2|<\left(R / \xi_{0}\right)^{2} G i_{(0)}$ is considered in the next subsection.

\section{Two-mode critical regime}

To obtain the expression for the critical fluctuations' contribution to paraconductivity at the magnetic flux $\phi \approx 1 / 2$ one should consider the dynamics of two lowest interacting modes. Further we restrict ourselves to the most interesting case when the magnetic flux through the ring is exactly half quantum $\phi=1 / 2$. In this case all modes except the modes with $n=0,1$ can be neglected since they do not contribute to the singular part of $\sigma_{0}$. Then the expression for $\sigma_{0}$ reads

$$
\begin{aligned}
& \sigma_{0}=\frac{1}{T}\left(\frac{e}{2 m R}\right)^{2} \\
& \times\left\langle\left(\left|\psi_{0}(0)\right|^{2}-\left|\psi_{1}(0)\right|^{2}\right) \int_{0}^{\infty}\left(\left|\psi_{0}(t)\right|^{2}-\left|\psi_{1}(t)\right|^{2}\right) d t\right\rangle .
\end{aligned}
$$

The system of nonlinear time-dependent GinzburgLandau equations for $\psi_{0}(t)$ and $\psi_{1}(t)$ is

$$
\left\{\begin{array}{l}
\gamma \partial_{t} \psi_{0}+\frac{\varepsilon^{*}}{4 m \xi_{0}^{2}} \psi_{0}+b\left(\left|\psi_{0}\right|^{2}+2\left|\psi_{1}\right|^{2}\right) \psi_{0}=0 \\
\gamma \partial_{t} \psi_{1}+\frac{\varepsilon^{*}}{4 m \xi_{0}^{2}} \psi_{1}+b\left(\left|\psi_{1}\right|^{2}+2\left|\psi_{0}\right|^{2}\right) \psi_{1}=0
\end{array}\right.
$$

where $\varepsilon^{*}=\varepsilon+\xi_{0}^{2} / 4 R^{2}$. Let us multiply the first equation of this system by $\psi_{0}^{*}$, the second equation by $\psi_{1}^{*}$ and then sum each of the obtained equations with its complex conjugated. The result is

$$
\left\{\begin{array}{l}
\partial_{\tilde{t}} F+a^{*} F+F^{2}+2 F G=0 \\
\partial_{\tilde{t}} G+a^{*} G+G^{2}+2 F G=0,
\end{array}\right.
$$

where $\tilde{t}=16 b t / \pi \alpha, a^{*}=\varepsilon^{*} / 4 m \xi_{0}^{2} b, F=\left|\psi_{0}\right|^{2}$ and $G=$ $\left|\psi_{1}\right|^{2}$. To simplify these equations we introduce a new time variable $\tau=\left(a^{*}\right)^{-1}\left[1-\exp \left(-a^{*} \tilde{t}\right)\right]$ and new functions $f(\tau)=e^{a^{*} \tilde{t}(\tau)} F(\tilde{t}(\tau))$ and $g(\tau)=e^{a^{*} \tilde{t}(\tau)} G(\tilde{t}(\tau))$. Then the system (13) transforms into the form

$$
\left\{\begin{array}{l}
\partial_{\tau} f+f^{2}+2 f g=0, \\
\partial_{\tau} g+g^{2}+2 f g=0 .
\end{array}\right.
$$

To obtain the exact solution of the system (14) it is convenient to consider the auxiliary functions $u=(f+$ $g) / 2$ and $v=(f-g) / 2$. Note that $\sigma_{0}$ depends only on $v(\tau)$ :

$$
\sigma_{0}=\frac{1}{T}\left(\frac{e}{m R}\right)^{2} \frac{\pi \alpha}{16 b}\left\langle v(0) \int_{0}^{1 / a^{*}} v(\tau) d \tau\right\rangle
$$

From the system (14) we obtain the equations for new functions

$$
\partial_{\tau} u=v^{2}-3 u^{2}, \quad \partial_{\tau} v=-2 u v,
$$

which can be reduced to the equation for the $v$ function

$$
2 v \partial_{\tau}^{2} v-5\left(\partial_{\tau} v\right)^{2}+4 v^{4}=0 .
$$

Then for the decaying solution of Eq. (17) we find

$$
\partial_{\tau} v=-2 \mu v^{2} \sqrt{1+\lambda v}
$$

where $\lambda=\left(u_{0}^{2}-v_{0}^{2}\right) / v_{0}^{3}$ (here and after the index 0 indicates the function value at $\tau=0$ and $v_{0}$ is assumed to be nonzero), $\mu=\operatorname{sign}\left(v_{0}\right)$.

Note that the explicit solution of the system (14) integrated over time has the form

$$
\begin{aligned}
& \int_{0}^{\infty} f(t) d t= \begin{cases}\ln \frac{2\left(f_{0}-g_{0}\right)^{2}}{f_{0} a^{*}} & \text { for } \mu=1, \\
\ln \frac{g_{0}}{\left|f_{0}-g_{0}\right|} & \text { for } \mu=-1\end{cases} \\
& \int_{0}^{\infty} g(t) d t= \begin{cases}\ln \frac{f_{0}}{\left|f_{0}-g_{0}\right|} & \text { for } \mu=1 ; \\
\ln \frac{2\left(f_{0}-g_{0}\right)^{2}}{g_{0} a^{*}} & \text { for } \mu=-1\end{cases}
\end{aligned}
$$




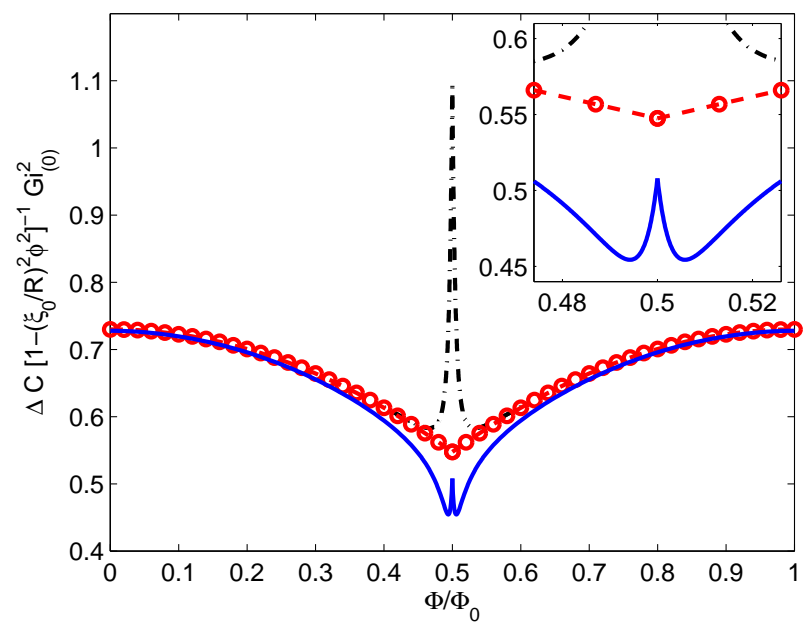

FIG. 1: (color online) The specific heat as a function of magnetic flux $\phi$ through the ring at the temperature of the superconducting transition for $\left(R / \xi_{0}\right)^{2} G i_{(0)}=10^{-2}$. The exact solution (blue solid curve) near $\phi=1 / 2$ qualitatively differs from the solution obtained taking only zero spatial mode into account (red dashed curve with circles) and is in sharp contrast with the predictions of the independent modes approximation (black dashdot curve).

An interesting feature of the solution (19) is that depending on the initial condition only one mode $(f$ or $g$ ) becomes slowly decaying due to the nonlinear modes' interaction.

The expression (18) allows us to calculate $\sigma_{0}$ since

$$
\int_{0}^{1 / a^{*}} v(\tau) d \tau=\int_{v_{0}}^{v\left(1 / a^{*}\right)} \frac{v}{\left(\partial_{\tau} v\right)} d v
$$

To estimate the $v\left(1 / a^{*}\right)$ value at the temperature of the superconducting transition $\left(a^{*} \rightarrow 0\right)$ we use the asymptotic form of the equation (18) solution, which gives us $v\left(1 / a^{*}\right) \approx \mu a^{*} / 2$. Then the singular part of the integral (18) has the form

$$
\int_{0}^{1 / a^{*}} v(\tau) d \tau \approx \frac{\left|v_{0}\right|}{2} \ln \frac{1}{\varepsilon^{*}}
$$

The Gibbs free energy functional for two considered modes has the form

$$
F=V\left[a^{*}\left(f_{0}+g_{0}\right)+\frac{b}{2}\left(f_{0}^{2}+g_{0}^{2}+4 f_{0} g_{0}\right)\right] .
$$

Performing the integration over $f_{0}$ and $g_{0}$ in the expression (15) in the limit $a^{*} \rightarrow 0$ we obtain

$$
\sigma_{0}(\phi=1 / 2)=\Lambda \frac{e^{2}}{\pi^{3 / 2} s^{2}}\left(\frac{\xi_{0}}{R}\right)^{4}\left(\frac{1}{2}\right)^{2} \frac{1}{G i_{(0)}^{3}} \ln \frac{1}{\varepsilon^{*}},
$$

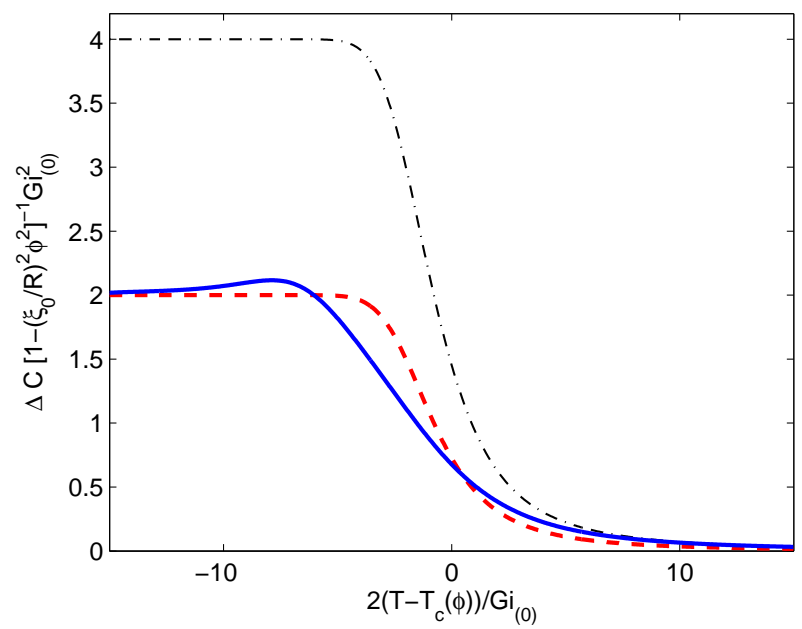

FIG. 2: (color online) The dependencies of the specific heat on temperature for $\phi=0$ (red dashed curve) and $\phi=1 / 2$ (blue solid curve). The black dashdot curve corresponds to the prediction of the independent modes approximation.

where $\Lambda=\pi(\sqrt{3}-1)[\sqrt{2} \operatorname{arcth}(\sqrt{2 / 3})]^{-1} \approx 1.42$. Thus at $\phi=1 / 2$ the exact expression of $\sigma_{0}$ is 1.42 times larger than the one obtained within the lowest mode approximation (see the expression (10)).

Note that for $\phi=1 / 2$ the nonzero components $\sigma_{k}$ do not diverge at $\varepsilon^{*}=0$. Indeed only the correlators in (4) that contain $\psi_{0}$ or $\psi_{1}$ functions can produce the singularity of $\sigma_{k}$ at $\varepsilon^{*}=0$. But the prefactor of the corresponding correlator in the $\sigma_{1}$ expression is zero in case $\phi=1 / 2$ and thus the $\sigma_{1}$ component does not contain the singular part. To calculate other $\sigma_{k}$ components we should take into account the following: (i) the correlators which contain noncritical modes can be represented as a product of two independent corelators; (ii) the corelators for critical modes can be calculated only for $\tau=0$ since near $\varepsilon^{*}=0$ the decay times $t_{n}=\pi R^{2} / 8 T \xi_{0}^{2} n(n-1)$ for noncritical modes are much shorter than for modes with $n=0,1$; (iii) for noncritical modes $\left\langle\psi_{n}(0) \psi_{n}^{*}(t)\right\rangle=\left\langle\left|\psi_{n}(0)\right|^{2}\right\rangle t_{n}$. Note also that for $\phi=1 / 2$ the correlators $\left\langle f_{0}\right\rangle=\left\langle g_{0}\right\rangle$. Therefore one can easily make sure that $\sigma_{k}$ does not contain a singular part at all since all contributions from critical modes cancel. Thus at $\phi=1 / 2$ only the $\sigma_{0}$ component has a singularity at $\varepsilon^{*}=0$.

It is interesting to analyze the conductivity behavior when the flux is slightly smaller than half-quantum $\delta \phi=$ $1 / 2-\phi \ll 1$. Then the system of equation (14) has the form

$$
\left\{\begin{array}{l}
\partial_{\tau} f+f^{2}+2 f g=0 \\
\partial_{\tau} g+\frac{\delta a^{*}}{\left(1-a^{*} \tau\right)} g+g^{2}+2 f g=0,
\end{array}\right.
$$

where $\delta a^{*}=\delta \phi /\left(2 m R^{2} b\right)$. Note that here the value $a^{*}$ which is used in the definition of functions $f$ and $g$ corresponds to modified critical temperature and reads as 
$a^{*}=\left(4 m \xi_{0}^{2}\right)^{-1}\left[\varepsilon+\left(\xi_{0} / R\right)^{2}(1 / 2-\delta \phi)^{2}\right]$. In what follows we will assume that $\delta \phi \ll\left(R / \xi_{0}\right)^{2} G i_{(0)}$.

For $f_{0}>g_{0}$ the dynamics of the slow-decaying mode $f$, which define the singular contribution to the conductivity, is weakly affected by the differences in the character of the mode $g$ decaying due to finite $\delta a^{*}$. At the same time for $g_{0}>f_{0}$ the situation changes. For the $\tau$ values when $g>>\delta a^{*} /\left(1-a^{*} \tau\right)$ the mode $g$ is slowly decaying while at $\tau \sim\left(a^{*}+\delta a^{*}\right)^{-1}$ which corresponds to the case $g \sim \delta a^{*} /\left(1-a^{*} \tau\right)$ the decaying of the $g$-mode becomes exponential. Thus for $g_{0}>f_{0}$ one has to replace the cutoff $1 / a^{*}$ in the integral (21) with $1 /\left(a^{*}+\delta a^{*}\right)$. Then the expression (23) becomes modified as follows

$$
\sigma_{0}(\delta \phi)=\frac{\Lambda e^{2}}{8 \pi^{3 / 2} s^{2}}\left(\frac{\xi_{0}}{R}\right)^{4} \frac{1}{G i_{(0)}^{3}}\left(\ln \frac{1}{\varepsilon^{*}}+\ln \frac{1}{\varepsilon^{*}+\delta \varepsilon^{*}}\right),
$$

where $\delta \varepsilon^{*}=2\left(\xi_{0} / R\right)^{2} \delta \phi$.

It is interesting to compare the expressions (11), (23) and (25). One can see that at magnetic fluxes close to $\phi=1 / 2$ the conductivity has rather special resonant structure. Indeed far from $\phi=1 / 2$ the conductivity is well described with the expression (10). When $\phi$ increases the conductivity becomes smaller compared with the expression (10) since the mode with $m=1$ begins to enter the critical regime. This picture is broken down when $1 / 2-\phi<<\left(R / \xi_{0}\right)^{2} G i_{(0)}$, where the logarithmic singularity in conductivity $\sigma_{0}(\phi)$ reveals a very sharp peak due to the nonlinear modes' interaction. The maximum of the peak at $\phi=1 / 2$ is larger than the one obtained in the lowest mode approximation (see the expression (10) ). The width of the peak is decreasing while $\varepsilon^{*} \rightarrow 0$ (see the expression (25)).

\section{FLUCTUATION SPECIFIC HEAT}

Now let us turn to the calculation of the fluctuation specific heat $\Delta C(\phi, T)$. The explicit form of the Gibbs energy $G[\psi]$ for two interacting modes with $n=0,1$ was obtained in Ref. 7. Then the specific heat can be calculated as $\Delta C=-T \partial_{T}^{2} G$. For $|\phi-1 / 2|>\left(R / \xi_{0}\right)^{2} G i_{(0)}$ the main contribution to the $\Delta C$ at $\varepsilon^{*} \rightarrow 0$ comes from the mode with $n=0$, which can be considered independently from other modes. Then

$$
\Delta C\left(T=T_{c}(\phi)\right)=\frac{2(\pi-2)}{\pi} \frac{1}{G i_{(0)}^{2}}\left(1-\frac{\xi_{0}^{2}}{R^{2}} \phi^{2}\right) .
$$

The corresponding dependence $\Delta C(\phi)$ at $\varepsilon^{*}=0$ is shown in Fig. 1 (red dashed curve with circles). Note that at $\phi=1 / 2$ the exact calculation gives the value $\Delta C=$ $\Theta G i_{(0)}^{-2}\left[1-\left(\xi_{0} / 2 R\right)^{2}\right]$, where $\Theta=(4 / 3)\left(1+\sqrt{3} \eta-\pi \eta^{2}\right)$, $\eta=(\operatorname{arcch} 2)^{-1}$.

Moreover it is interesting to analyze the dependence of the specific heat on temperature. In Fig. 2 two typical dependencies $\Delta C(T)$ are shown for $\phi=0$ (red dashed curve) and for $\phi=1 / 2$ (blue solid curve). In addition to the differences between plotted curves near the critical temperature one can see that in the superconducting state at $\phi=1 / 2$ the $\Delta C$ has shallow peak due to the interaction of two critical modes.

Note that the exact solution for the specific heat in two-modes critical regime is in a sharp contrast with the predictions obtained within the independent modes approximation. For comparison in Fig. 1 and 2 we have plotted corresponding dependencies calculated without considering the modes' interaction (black dashdot curves). One can see that these curves differ qualitatively from the ones corresponding to exact solutions.

\section{CONCLUSION}

Thus we described the critical fluctuations in the regime when two interacting modes are strongly fluctuating. Both specific heat and magnetoconductivity are reveal special resonant behavior for magnetic flux $\phi \approx 1 / 2$, which may serve as clear indication of the two-mode critical regime for eventual experiments. Note that our results obtained for superconducting ring can be generalized directly for superconducting disk ${ }^{16}$ or the superconducting layer with the hole of the round shape $\frac{17}{}$. In these systems the magnetic field which exceeds the upper critical field $H_{c 2}$ but is less than the field of the surface superconductivity $H_{c 3}$ leads to appearance of the superconducting nucleus localized in the ring with the width of the order of $\xi_{0}$ near the superconductor edge.

The predicted two modes critical fluctuations regime produces a relatively strong variation of magnetoconductivity and specific heat (of the order of $30 \%-100 \%$ near the half flux field). The first detailed measurements of the paraconductivity in small superconducting loops have been reported already in Ref. 11, and recently the precise contactless studies of the magnetic response in individual normal metal rings have been performed ${ }^{20}$. Therefore the experimental studies of the critical regime in magnetoconductivty seem to be quite feasible. Five years ago the sensitive attojoule calorimetry $\underline{\underline{18}, 19}$ revealed the specific heat oscillations in magnetic field for large arrays (450 thousands) of noninteracting micrometer-sized superconducting loops. In such experiments the averaging effects may play an important role. Unfortunately the statistical characteristics of the studied ensemble of loops are unknown, which does not allow comparing their results with our calculations. However the recent progress in this domain should permit to perform the calorimetric measurements on a single superconducting ring in near future.

\section{ACKNOWLEDGEMENTS}

The authors thank M. Daumens, A. Varlamov and A. Mel'nikov for many useful discussions and sugges- 
tions. This work was supported by the European IRSES program SIMTEC, French ANR "SINUS", the RFBR, Presidential RSS Council (Grant No.MK-4211.2011.2), RAS under the Program "Quantum physics of condensed matter", the "Dynasty" Foundation and FTP "Scientific and educational personnel of innovative Russia in 2009$2013 "$.

\section{APPENDIX}

In the Gaussian regime one can calculate the part of the conductivity which can become singular near the temperature of the superconducting transition for $0 \leq \phi \leq 1 / 2$, considering only terms with $m=0$ and $m=1$ in the expression (3). Then using the fact that in the Gaussian regime for $k \neq l$ the identity $\left\langle\left|\psi_{k}(0)\right|^{2}\left|\psi_{l}(0)\right|^{2}\right\rangle=\left\langle\left|\psi_{k}(0)\right|^{2}\right\rangle\left\langle\left|\psi_{l}(0)\right|^{2}\right\rangle$ is true and that $\int_{0}^{\infty} \exp \left(-t / \tau_{n}\right) d t=\tau_{n}$ we obtain

$$
\begin{array}{r}
\sigma_{0}=\frac{1}{T}\left(\frac{e}{m R}\right)^{2}\left[\phi^{2} \tau_{0}\left\langle\left|\psi_{0}(0)\right|^{4}\right\rangle-\phi \tau_{0}\left\langle\left|\psi_{0}(0)\right|^{2}\right\rangle \sum_{n \neq 0}(n-\phi)\left\langle\left|\psi_{n}(0)\right|^{2}\right\rangle\right. \\
\left.+(1-\phi)^{2} \tau_{1}\left\langle\left|\psi_{1}(0)\right|^{4}\right\rangle+(1-\phi) \tau_{1}\left\langle\left|\psi_{1}(0)\right|^{2}\right\rangle \sum_{n \neq 1}(n-\phi)\left\langle\left|\psi_{n}(0)\right|^{2}\right\rangle\right] .
\end{array}
$$

Substituting the expressions (6) and (7) into (27) we obtain

$$
\begin{array}{r}
\sigma_{0}=\frac{1}{T}\left(\frac{e}{m R}\right)^{2} \frac{\left(4 m \xi_{0}^{2} T\right)^{2}}{V^{2}}\left[\frac{2 \phi^{2} \tau_{0}}{\left(\varepsilon^{*}\right)^{2}}-\frac{\phi \tau_{0}}{\varepsilon^{*}} \sum_{n \neq 0} \frac{(n-\phi)}{\left[\varepsilon^{*}+\left(\xi_{0} / R\right)^{2} n(n-2 \phi)\right]}\right. \\
\left.+\frac{2(1-\phi)^{2} \tau_{1}}{\left[\varepsilon^{*}+\left(\xi_{0} / R\right)^{2}(1-2 \phi)\right]^{2}}+\frac{(1-\phi) \tau_{1}}{\left[\varepsilon^{*}+\left(\xi_{0} / R\right)^{2}(1-2 \phi)\right]} \sum_{n \neq 1} \frac{(n-\phi)}{\left[\varepsilon^{*}+\left(\xi_{0} / R\right)^{2} n(n-2 \phi)\right]}\right] .
\end{array}
$$

To calculate the principal values of the sums in the expression (28) near the critical temperature one can put $\varepsilon^{*}=0$ in all terms except those with $n=0,1$. The results are

$$
\begin{gathered}
\sum_{n \neq 0} \frac{(n-\phi)}{\left[\varepsilon^{*}+\left(\xi_{0} / R\right)^{2} n(n-2 \phi)\right]}= \\
=\frac{(1-\phi)}{\varepsilon^{*}+\left(\xi_{0} / R\right)^{2}(1-2 \phi)}-\frac{(1+\phi)}{\left(\xi_{0} / R\right)^{2}(1+2 \phi)}+\sum_{n \geq 2}\left[\frac{(n-\phi)}{\left(\xi_{0} / R\right)^{2} n(n-2 \phi)}-\frac{(n+\phi)}{\left(\xi_{0} / R\right)^{2} n(n+2 \phi)}\right]= \\
=\frac{(1-\phi)}{\varepsilon^{*}+\left(\xi_{0} / R\right)^{2}(1-2 \phi)}-\frac{(1+\phi)}{\left(\xi_{0} / R\right)^{2}(1+2 \phi)}+2 \phi\left(\frac{R}{\xi_{0}}\right)^{2}\left[\frac{1-12 \phi^{2}}{8 \phi^{2}\left(1-4 \phi^{2}\right)}-\frac{\pi}{4 \phi} \operatorname{ctg}(2 \pi \phi)\right]
\end{gathered}
$$

and

$$
\sum_{n \neq 1} \frac{(n-\phi)}{\left[\varepsilon^{*}+\left(\xi_{0} / R\right)^{2} n(n-2 \phi)\right]}=-\frac{\phi}{\varepsilon^{*}}-\frac{(1+\phi)}{\left(\xi_{0} / R\right)^{2}(1+2 \phi)}+\left(\frac{R}{\xi_{0}}\right)^{2}\left[\frac{1-12 \phi^{2}}{4 \phi\left(1-4 \phi^{2}\right)}-\frac{\pi}{2} \operatorname{ctg}(2 \pi \phi)\right] .
$$

Summarizing we obtain the final result for the singular part of the component $\sigma_{0}^{(G)}$ in the Gaussian regime:

$$
\begin{gathered}
\sigma_{0}^{(G)}\left(\phi, \varepsilon^{*}\right)=\frac{e^{2}}{4 \pi s^{2}}\left(\frac{\xi_{0}}{R}\right)^{4} \frac{\phi^{2}}{\left(\varepsilon^{*}\right)^{3}}+\frac{e^{2}}{2 \pi s^{2}}\left(\frac{\xi_{0}}{R}\right)^{4} \frac{(1-\phi)^{2}}{\left[\varepsilon^{*}+\left(\xi_{0} / R\right)^{2}(1-2 \phi)\right]^{3}}- \\
-\frac{e^{2}}{4 \pi s^{2}}\left(\frac{\xi_{0}}{R}\right)^{2} \frac{\phi}{\left(\varepsilon^{*}\right)^{2}}\left[\left(\frac{\xi_{0}}{R}\right)^{2} \frac{(1-\phi)}{\left[\varepsilon^{*}+\left(\xi_{0} / R\right)^{2}(1-2 \phi)\right]}-\frac{(1+\phi)}{(1+2 \phi)}+\left(\frac{1-12 \phi^{2}}{4 \phi\left(1-4 \phi^{2}\right)}-\frac{\pi}{2} \operatorname{ctg}(2 \pi \phi)\right)\right]+ \\
+\frac{e^{2}}{4 \pi s^{2}}\left(\frac{\xi_{0}}{R}\right)^{2} \frac{(1-\phi)}{\left[\varepsilon^{*}+\left(\xi_{0} / R\right)^{2}(1-2 \phi)\right]^{2}}\left[-\left(\frac{\xi_{0}}{R}\right)^{2} \frac{\phi}{\varepsilon^{*}}-\frac{(1+\phi)}{(1+2 \phi)}+\left(\frac{1-12 \phi^{2}}{4 \phi\left(1-4 \phi^{2}\right)}-\frac{\pi}{2} \operatorname{ctg}(2 \pi \phi)\right)\right] .
\end{gathered}
$$


For magnetic flux values which are far from the point $\phi=1 / 2$ the analogous singular part of the conductivity in the critical regime can be calculated under the assumption that the fluctuations of only the mode with $m=0$ are critical while the fluctuations of other spatial modes are Gaussian (the lowest mode approximation). The procedure of calculation is similar to the one for the Gaussian regime. The expression (3) takes the form

$$
\begin{aligned}
\sigma_{0}=\frac{1}{T}\left(\frac{e}{m R}\right)^{2} & {\left[\phi^{2}\left\langle\left|\psi_{0}(0)\right|^{2} \int_{0}^{\infty}\left|\psi_{0}(t)\right|^{2} d t\right\rangle-\phi\left\langle\int_{0}^{\infty}\left|\psi_{0}(t)\right|^{2} d t\right\rangle \sum_{n \neq 0}(n-\phi)\left\langle\left|\psi_{n}(0)\right|^{2}\right\rangle\right.} \\
& \left.+(1-\phi)^{2} \tau_{1}\left\langle\left|\psi_{1}(0)\right|^{4}\right\rangle+(1-\phi) \tau_{1}\left\langle\left|\psi_{1}(0)\right|^{2}\right\rangle \sum_{n \neq 1}(n-\phi)\left\langle\left|\psi_{n}(0)\right|^{2}\right\rangle\right] .
\end{aligned}
$$

The first term in the square brackets in the expression (32) represents the central result of Ref. 13. To calculate the second term one should take into account that in the critical regime

$$
\left\langle\int_{0}^{\infty}\left|\psi_{0}(t)\right|^{2} d t\right\rangle=\frac{m \xi_{0}^{2}}{4 R s} \frac{1}{G i_{(0)}^{2}} \ln \frac{1}{\varepsilon^{*}} .
$$

The last sum in the square brackets in the expression (32) is similar to the one in the expression (27) except the term with $n=0$, which should be calculated in the critical regime. The expression for the corresponding correlator in the explicit form is given in Ref. 13. Summarizing we obtain the resulting expression for the conductivity in the critical regime for magnetic flux values which are far from $\phi=1 / 2$ :

$$
\begin{gathered}
\sigma_{0}^{(C)}(\phi)=\frac{e^{2}}{\pi^{3 / 2} s^{2}}\left(\frac{\xi_{0}}{R}\right)^{4} \phi^{2} \frac{1}{G i_{(0)}^{3}} \ln \frac{1}{\varepsilon^{*}}+\frac{e^{2}}{2 \pi s^{2}}\left(\frac{\xi_{0}}{R}\right)^{4} \frac{(1-\phi)^{2}}{\left[\varepsilon^{*}+\left(\xi_{0} / R\right)^{2}(1-2 \phi)\right]^{3}}- \\
-\frac{e^{2}}{2 \pi s^{2}} \phi\left(\frac{\xi_{0}}{R}\right)^{2} \frac{1}{G i_{(0)}^{2}} \ln \frac{1}{\varepsilon^{*}}\left[\left(\frac{\xi_{0}}{R}\right)^{2} \frac{(1-\phi)}{\left[\varepsilon^{*}+\left(\xi_{0} / R\right)^{2}(1-2 \phi)\right]}-\frac{(1+\phi)}{(1+2 \phi)}+\left(\frac{1-12 \phi^{2}}{4 \phi\left(1-4 \phi^{2}\right)}-\frac{\pi}{2} \operatorname{ctg}(2 \pi \phi)\right)\right]- \\
-\frac{e^{2}}{2 \pi^{3 / 2} s^{2}}\left(\frac{\xi_{0}}{R}\right)^{6} \frac{1}{G i_{(0)}} \frac{\phi(1-\phi)}{\left[\varepsilon^{*}+\left(\xi_{0} / R\right)^{2}(1-2 \phi)\right]^{2}} .
\end{gathered}
$$

Thus the expressions (31) and (34) give the singular part of the conductivity $\sigma_{0}$ in the Gaussian and critical (in the lowest mode approximation) regimes respectively.

1 W. A. Little and R. D. Parks, Phys. Rev. Lett. 9, 9 (1962)

2 N. C. Koshnick, H. Bluhm,M. E. Huber, K. A. Moler, Science 318, 1440 (2007)

3 M. Morelle, D. S. Golubovic and V. Moshchalkov, Phys. Rev. B 70, 144528 (2004)

4 A. A. Abrikosov, Fundamentals of Theory of Metals (North-Holland, Amsterdam, 1988)

${ }^{5}$ G. Schwiete and Y. Oreg, Phys. Rev. Lett. 103, 037001 (2009)

${ }^{6}$ G. Schwiete and Y. Oreg, Phys. Rev. B 82, 214514 (2010)

7 M. Daumens, C. Mayers, A. Buzdin, Phys. Lett. A 248, $445(1998)$

$8 \mathrm{~V}$. V. Schmidt, in Proceedings of the 10th International Conference on Low Temperature Physics (VINITI, Moscow), C2, 205 (1967)
${ }^{9}$ R. A. Buhrman and W. P. Halperin, Phys. Rev. Lett. 30, 692 (1973)

10 L. G. Aslamazov and A. I. Larkin, Phys. Lett. A 26, 238 (1968).

11 N. E. Israeloff, F. Yu, A. M. Goldman and R. Bojko, Phys. Rev. Lett. 71, 2130 (1993)

12 J. S. Langer and V. Ambegaokar, Phys. Rev. 164, 498 (1967)

13 A. I. Buzdin and A. A. Varlamov, Phys. Rev. Lett. 89, 076601 (2002)

14 L. I. Glazman, F. W. J. Hekking and A. Zyuzin, Phys. Rev. B 46, 9074 (1992)

15 A. I. Larkin and A. A. Varlamov, in Physics of Conventional and Nonconventional Superconductors, edited by K.-H. Bennemann and J. B. Ketterson (Springer-Verlag, 
Berlin, 2002)

16 O. Buisson, P. Gandit, R. Rammal, Y. Y. Wang and B. Pannetier, Phys. Lett. A 150, 36 (1990)

17 A. Bezryadin, A. Buzdin and B. Pannetier, Phys. Rev. B 51, 3718 (1995)

18 O. Bourgeois, S. E. Skipetrov, F. Ong, and J. Chaussy,
Phys. Rev. Lett. 94, 057007 (2005)

19 F. R. Ong, O. Bourgeois, S. E. Skipetrov, and J. Chaussy, Phys. Rev. B 74, 140503(R) (2006)

${ }^{20}$ H. Bluhm, N. C. Koshnick, J. A. Bert, M. E. Huber, and K. A. Moler, Phys. Rev. Lett. 102, 136802 (2009). 